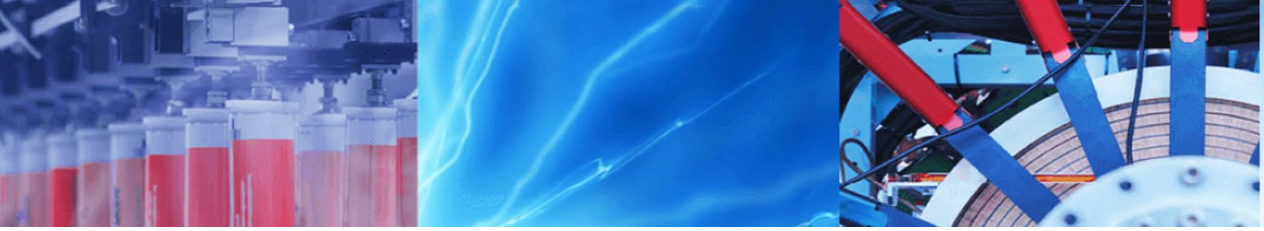

Research Article

\title{
MRI denoising using BM3D equipped with noise invalidation denoising technique and VST for improved contrast
}

\author{
V. Hanchate ${ }^{1} \cdot$ K. Joshi ${ }^{2}$
}

Received: 18 October 2019 / Accepted: 3 January 2020 / Published online: 18 January 2020

(c) Springer Nature Switzerland AG 2020

\begin{abstract}
Denoising of medical scanned images such as X-ray, MRI etc. is important stage in the medical use. To remove the noise from "magnetic resonance images" (MRI) is the attention of researchers to generate the MR images with high "signalto-noise ratio" as well as with high spatial resolution. In this denoising technique, block-matching and 3-dimensional filtering (BM3D) method is used to denoise the MR images. Main steps used in BM3D are grouping, 3-dimensional discrete wavelet transformation and wavelet shrinkage. In the proposed method, noise invalidation denoising technique (NIDe) is used rather than hard thresholding. NIDe gives the threshold value automatically based on the data and noise characteristics and threshold value changes according to the characteristics of data i.e. wavelet coefficient of image. Before denoising MR images, variance stabilization transform (VST) discard the noise variance dependency of the MRI intensities. Combining block-matching and 3-dimensional filtering technique and VST make able the use of the BM3D technique for Magnetic Resonance Image denoising. After BM3D i.e. final denoised MR image, "contrast limited adaptive histogram equalization" technique is applied to increase the contrast of MR images which are denoised. Performance metrics such as "Peak Signal to Noise ratio", "Root Mean Square Error", "Mutual Information", "Edge Entropy" and "Structural Similarity Index Method" are found out for "T1 weighted", "T2 weighted" and "PD weighted" magnetic resonance images.
\end{abstract}

Keywords Block-matching and 3D filtering · Magnetic resonance imaging · Denoising · Noise invalidation denoising · Variance stabilization transform $\cdot$ Contrast limited adaptive histogram equalization

\section{Introduction}

Need of medical imaging modalities is proper diagnosis of various disease. Different medical imaging modalities are developed for different disease diagnosis since last few decades. Medical imaging modalities are used to generate the images of internal structures in the body and this anatomical structure is checked from these images rather than opening the body. Now a day's, Nuclear Imaging, Ultrasound, "X-rays", "Computed Tomography" scan and "Magnetic Resonance Imaging" are the mostly used medical imaging. However all these medical imaging modalities have one drawback i.e. presence of noise. When the true pixel value is replaced by some faulty value, then pixel is said to be noisy.

Noise can be introduced in the image while acquiring the image or during transmission; different image modality can suffer from different types of noise such as "QUANTUM noise" is present in "X-rays" and "Nuclear imaging". Also, "speckle noise" is present in "ultrasound imaging" and Rician distribution in "Magnetic resonance imaging" etc. This various noises in the medical images also reduces the contrast of these images. Therefore, disease diagnosis becomes a very difficult task. So that it is required to denoise the medical images [1].

$\triangle$ V. Hanchate, vnjpune@gmail.com; K. Joshi, krjpune@gmail.com | 'Department of E\&TC, College of Engineering, Pune, India. ${ }^{2}$ Department of E\&TC, PES's MCOE, Pune, India. 
MR images is medical imaging technique which scans tissues in the human body. "MRI system" is mainly working on the objective of "nuclear magnetic resonance (NMR)". It uses the interaction of "electromagnetic field" and "nuclean spin" to map the properties and spatial location of tissues. When hydrogen atom comes in contact with a strong magnetic field and it is excited by an external magnetic filed; it generates the spin which is detected and processed by the MR system. The human body is largely composed of water molecules and fat. Water molecules have two hydrogen protons. These hydrogen protons are mainly imaged to demonstrate the pathological and physiological changes of tissues in the human body [2]. Acquisition time in magnetic resonance imaging (MRI) is very less. Therefore, signal to noise ratio (SNR) value of the magnetic resonance imaging are generally low. The qualities of the MR images are generally degraded with various noises and different artifact which is modeled as "Rician" distribution. Denoising methods that discards the noisy coefficients and preserves the main information in the MR images is an important stage in image processing [3].

Now a day's large improvement is done in the medical imaging technology considering SNR value and spatial resolution. Still MR images are affected by the noise and artifacts. Magnetic resonance images mainly consist of "thermal noise" that is generated from the "scanned object".

\subsection{Characteristic of noise in MR images}

The raw data is generated at the time of "MRI scanning" which is complex values correspond to the discrete "Fourier transform (DFT)" of a magnetization distribution of a volume of tissues. Therefore, MR image is reconstructed by computing the IDFT of the raw data. IDFT converts this data into magnitude, frequency and phase components that represent the morphological and physiological features in the body of who is scanned. Signal components are available in the real channel as well as in imaginary channel. Both channels are influenced by AWGN. Magnitude of MR Image is calculated as square root of the sum of squares of the two independent "Gaussian variables". Therefore, it tends to "Rician distribution". In low intensity regions i.e. in dark region, the "Rician distribution" follows "Rayleigh distribution" and in high intensity i.e. in bright regions it follows "Gaussian distribution" [4].

$p_{M}\left(M \mid A, \sigma_{n}\right)=\frac{M}{\sigma_{n}^{2}} e^{-\left(M^{2}+A^{2}\right) / 2 \sigma_{n}^{2}} I_{0}\left(\frac{A M}{\sigma_{n}^{2}}\right) u(M)$

where $I_{0}$ is the modified zeroth order "Bessel function" of the first kind, $\sigma_{n}^{2}$ is the "noise variance", A the noiseless signal level, magnitude variable is $\mathrm{M}$ and $\mathrm{u}($.$) is the "Heavi-$ side step function".

\section{Related work}

Figure 1 shows an ample of denoising techniques discussed in the past and these techniques have some advantages, drawbacks and future scope [4]. The purpose of this literature survey is to present a review of the published paper in dealing with denoising methods in "MR images". Spatial and temporal filters discussed by McVeigh et al. [5] minimize the Gaussian noise. But the noise present in MRI is Rician. Anisotropic diffusion filter (ADF) is presented by Krissian and Aja-Fernandez [6] to remove noise from magnetic resonance imaging. "Non local mean filter" is presented by Buades et al. [7] to remove the noise from natural images. MR images are denoised by Manjon et al. [8] using the unbiased "Non Local Means" filter approach. Non iterative alternative to anisotropic diffusion filter (ADF) is provided by Tomasi and Manduchi [9] by presenting the bilateral filter. Noise reduction in medical images is presented by Wong et al. [10,11]. Concept of wavelet shrinkage is proposed by Yu and Zhao [12] for removal of "Rician noise" from "magnetic resonance image". BM3D is post acquisition based noise reduction method. Block matching and 3-dimensional filtering (BM3D) technique has advantages in denoising images with "additive white Gaussian noise (AWGN)".

\section{Proposed BM3D NIDE VST method}

BM3D is one of the MRI denoising technique. Block matching and 3-dimensional filtering technique consist of two main steps (Fig. 2).

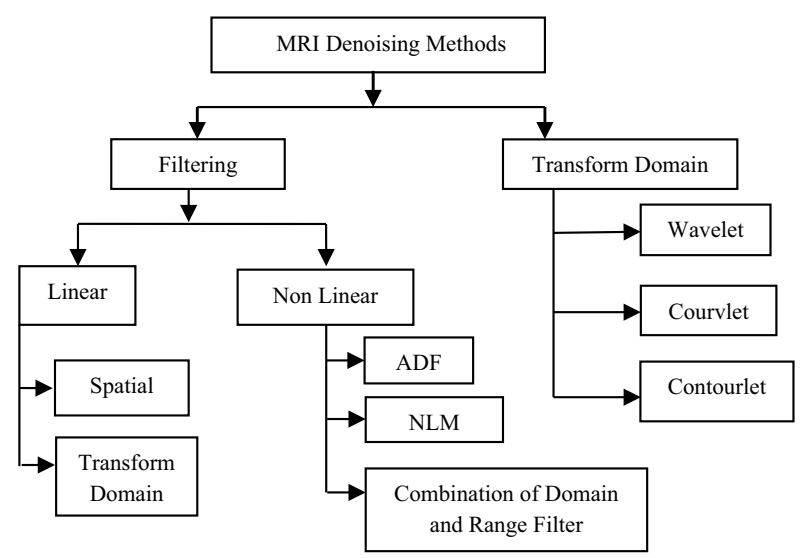

Fig. 1 Classification of MRI denoising methods 


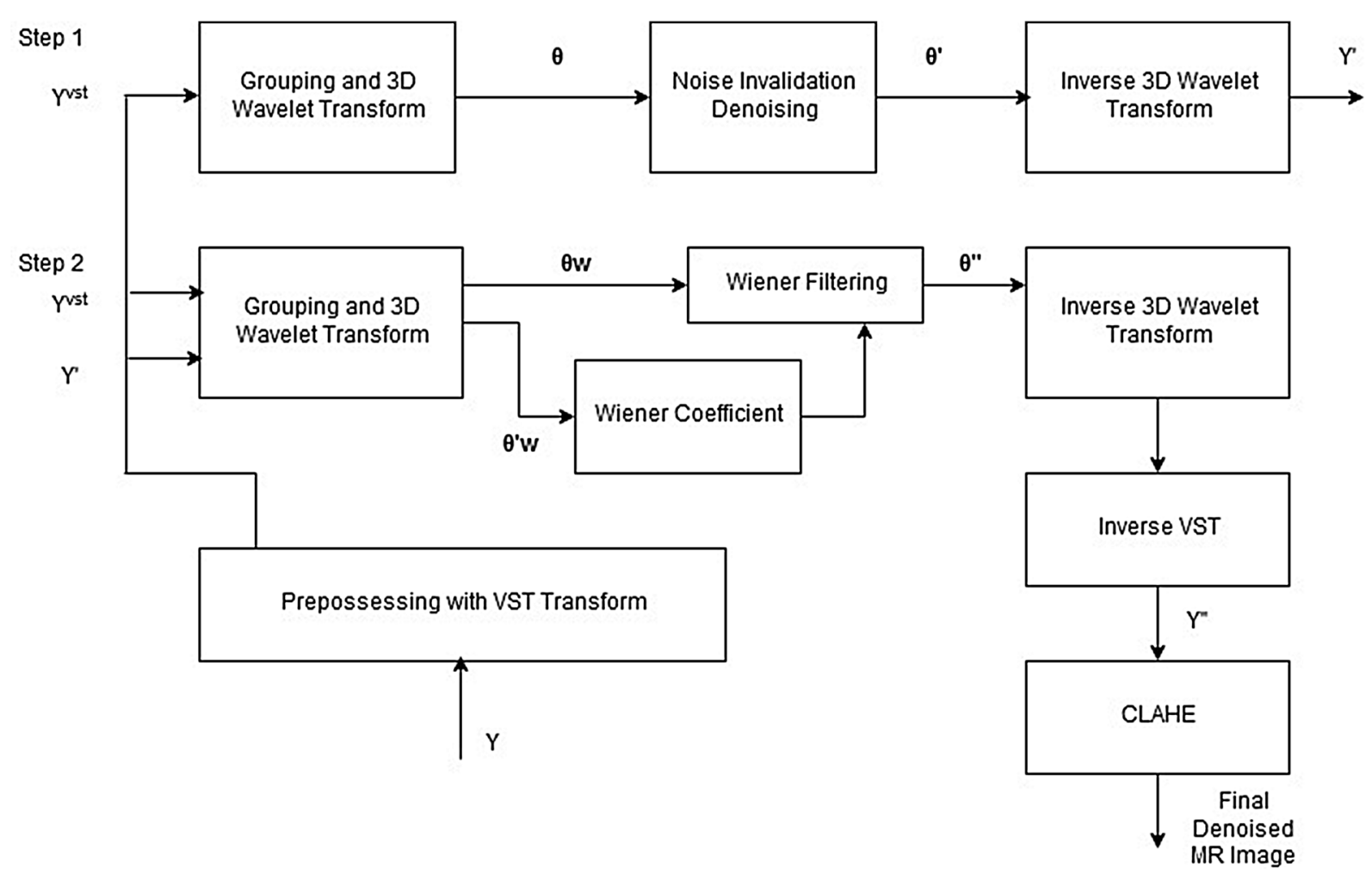

Fig. 2 Block diagram of proposed method

- Denoise the MR image by using NIDe

- Denoise the MR image by using Wiener filtering collaborative filtering is used in these two steps.

Each step consists of four stages [2]:

- Grouping patches which are same as reference patch

- 3-Dimensional discrete wavelet transformation of each group

- Denoising wavelet coefficients using NIDe in first step and using Wiener Filtering in the second step

- Inverse 3-dimensional discrete wavelet transformation.

Block matching and 3-dimensional filtering is enhanced technique of Non Local Mean filtering. If we apply the discrete wavelet transformation on the similar group of patches, it increases the sparsity of the data as compared to applying transformation on original image. So, BM3D can remove the noise easily $[13,14]$.

The main purpose of block matching and 3-dimensional filtering is to remove noise from the group of similar patches using Wiener filter. But, Wiener filtering needs estimation of group of the similar patches. This estimation is found in the first step of block matching and 3-dimensional filtering which consist of 3-dimensional discrete wavelet transformation and NIDe for thresholding purpose on the group of similar patches which is generated by the original noisy image. This first step estimation is used in the second step of block matching and 3-dimensional filtering which finds the group of similar patches for the Wiener filtering step. For thresholding purpose, NIDe method is proposed instead of the hard thresholding. Block matching 3-dimensional filtering technique is used in denoising MR images only if images are preprocessed by VST method $[15,16]$, which removes the dependency of "noise variance" in the "Rician" distributed magnetic resonance images.

\subsection{Preprocessing with VST method}

The boundaries of noise in noise invalidation denoising techniques are represented according to AWGN, but the Rician distribution present in the MRI is not equivalent to the zero mean. Also, noise variance is dependent on the image intensity in Rician distribution. Therefore, we cannot apply BM3D method directly on MRI [14]. Variance stabilization transformation reduces the bias and also transforms the Rician distribution to Gaussian by stabilizing noise variance. Therefore VST approach is mainly used in proposed BM3D NIDe VST algorithm. Final denoised MR image is 
generated by performing an inverse variance stabilization transformation to the denoised image.

Anscombe transform [17] is mostly used VST and considered to be a useful tool due to its simplicity and efficiency. It's expression is as given below.

$Y_{i}=T\left(y_{i}\right)=2 \sqrt{y_{i}+\frac{3}{8}}$

where $y_{i}$ is the observed intensity value of noisy image and $Y_{i}$ is the transformed intensity value. After the anscombe transformation $\mathrm{T}$, the pixel intensities throughout the whole image are approximately "Gaussian distributed" with mean 0 and "variance" $\sigma^{2}=1$. Thus its variance is assumed to be stationary.

\subsection{Representation of noisy image}

Consider 2-D gray image $\{\bar{Y}(x) \mid x \in \Omega\}$ which is represented in a spatial domain $\Omega \subset R^{2}$, where $x$ is the coordinate of each pixel in the image. This image is corrupted with an AWGN having mean value 0 and variance $\sigma^{2}$. The noisy image $y$ is defined as:

$y(x)=\bar{y}(x)+w(x)$

To remove effect of $w(x)$ is the objective of BM3D [3].

\subsection{Grouping of similar patches and 3-dimensional DWT}

"Grouping" is defined as collection of similar d- dimensional patches of an image into $(d+1)$-dimensional structure. In 2-dimensional MR images, patches are 2-dimensional signal (arbitrary 2-D neighborhoods). First we select one reference patch. According to that reference patch, find out all the similar patches and group them together.
In the same manner for different reference patches, find out the different groups. In this way grouping is a 3-dimensional signal produced by combining similar 2-D patches together (image neighborhoods) (Fig. 3).

The main aim of grouping is utilization of a higher dimensional filtering, which exploit the similarity between groups which consist of similar patches to find out the original signal. Similarity between all the patches of image is find out according to the distance between patches i.e. less distance shows high similarity [13]. Here, $I_{p}$-norm formula is used to find out distance which is given as,

$\left\|x_{1}-x_{2}\right\|_{2}=\sqrt{\sum_{i}\left(x_{1 i}-x_{2 i}\right)^{2}}$

After the grouping (i.e. 3-D array), apply 3-D DWT on the entire 3-D array and get wavelet coefficient.

\subsection{Denoising wavelet coefficient using NIDe and Wiener filtering}

The denoising stages denoise following coefficients of the noisy image:

$\theta(x)=\bar{\theta}(x)+v(x)$

where $\bar{\theta}(x)$ are noiseless coefficients and $v(x)$ are noisy coefficients. In the first step, wavelet coefficients of MR images are denoised by using NIDe instead of hard thresholding. In hard thresholding, threshold value is find out according to the trial and error method considering large number of samples. This method is very complex and replaced by NIDe method. NIDe denoises the coefficient adaptive to the noisy image. Threshold value is find out automatically according to the data and noise characteristics [18]. NIDe method removes the coefficient
Fig. 3 General example of grouping

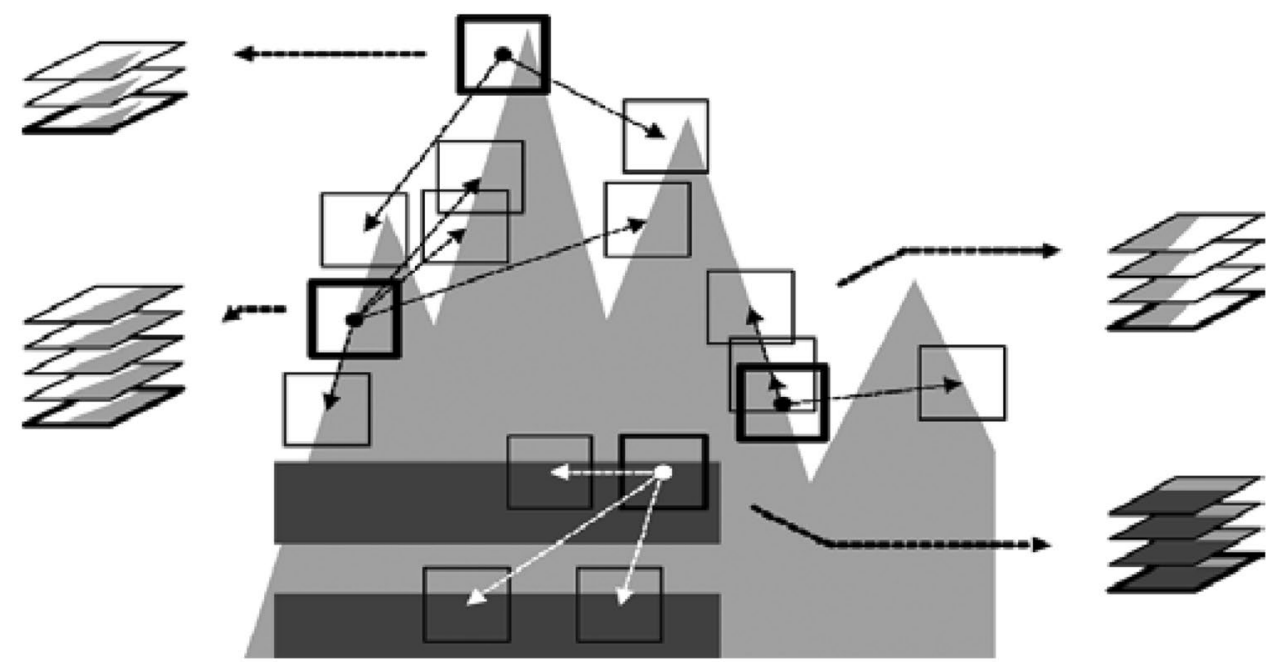


of noisy image that follows the statistics associated with the AWGN. After by applying inverse 3D DWT, we get estimated image.

Let us denote the $\theta$ coefficients in (5) as ' $z$ '.

$\theta \rightarrow z$

Noisy coefficient of $z$, represented as $n_{z}$. Mean and variance of this noisy coefficient is

$E\left(n_{z}\right)=F\left(n_{z}\right)$

$\operatorname{var}\left(n_{z}\right)=\frac{1}{n} F\left(n_{z}\right)\left(1-F\left(n_{z}\right)\right.$

where $F(\cdot)$ is the available "cumulative distribution function (CDF)" of AWGN $v$ in Eq. (5) and $N$ is the total number of pixels in the image. $n_{z}$ represents noise signature for the noise invalidation purpose i.e. any coefficient of $z$ which isinside the boundary of $E\left(n_{z}\right)+/-\lambda \sqrt{\operatorname{var}\left(n_{z}\right)}$ is considered as noise and these coefficients are removed. The $\lambda$ is chosen based on the probabilistic confidence of the normal distribution CDF. Applying IDWT, we get estimated denoised image. This estimated image and VST processed image is used in the second step as input for wiener filtering. Considering a wiener filter in frequency domain as $(u$, v). Restored image will be given as;

$X(u, v)=G(u, v) \cdot W(u, v)$

where $W(u, v)$ is the received signal and $X(u, v)$ is the restored image.
Same procedure is repeated in the second step explained above. Difference is that Wiener filtering is used instead of NIDe. By applying inverse 3D DWT at the end of second stage, we get denoised MR image. Inverse VST is applied on this denoised MR image to get final denoised MR image (Fig. 4).

\subsection{Contrast limited adaptive histogram equalization (CLAHE)}

If the medical images are of low contrast then it is difficult for doctors to analyze them. Therefore, it is required to increase the image contrast. This can be done by number of ways in image processing. CLAHE is mainly used for enhancement of MR images. After second step output of BM3D i.e. final denoised MR image, apply CLAHE to increase the contrast of denoised MR image. CLAHE is applied on small regions of image data instead of entire image. The resulting neighboring tiles are then stitched back using bilinear interpolation technique. The contrast of image in the homogeneous region can be limited so that noise amplification can be avoided. While performing AHE (Adaptive Histogram Equalization) noise level is increased if the region has very small intensity range. To remove this drawback in $\mathrm{AHE}$, some changes are done in this method called CLAHE [19].

Fig. 4 Block diagram of Wiener filter

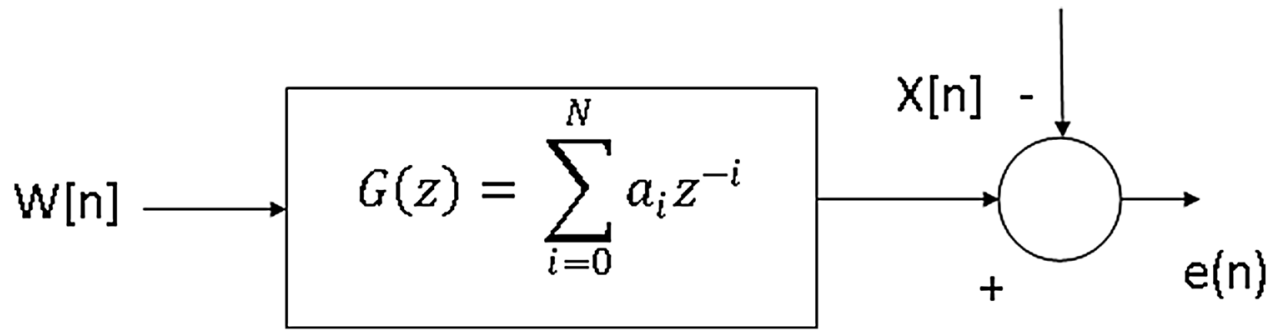

Table 1 Results for different MRI for different noise level

\begin{tabular}{lccccccc}
\hline & $3 \%$ & $5 \%$ & $9 \%$ & $11 \%$ & $13 \%$ & $15 \%$ & $17 \%$ \\
\hline PSNR & 43.014 & 40.251 & 35.264 & 33.402 & 31.371 & 29.804 & 28.034 \\
RMSE & 0.4993 & 0.4993 & 0.4994 & 0.4994 & 0.4994 & 0.4995 & 0.4996 \\
SSIM & 0.9979 & 0.9978 & 0.9875 & 0.9872 & 0.9868 & 0.9867 & 0.9765 \\
MI & 1.1296 & 0.9746 & 0.7295 & 0.6446 & 0.5658 & 0.5113 & 0.4581 \\
Edge intensity & 2.7574 & 2.6993 & 2.6525 & 2.5842 & 2.5354 & 2.42562 & 2.3685 \\
Time & 546.54 & 381.95 & 309.21 & 272.34 & 261.68 & 206.443 & 201.52 \\
\hline
\end{tabular}




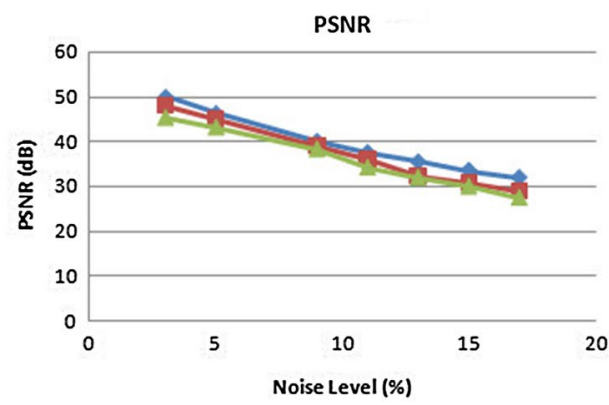

(a)

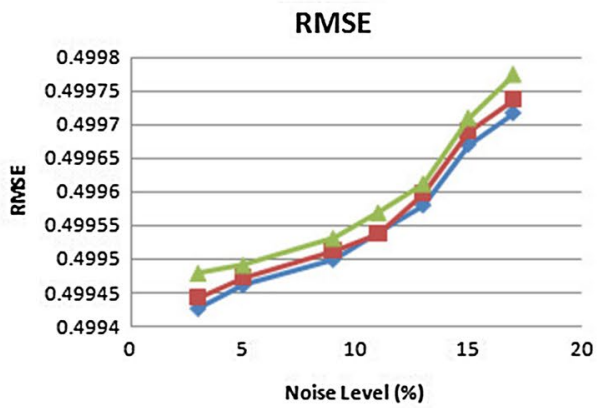

(b)

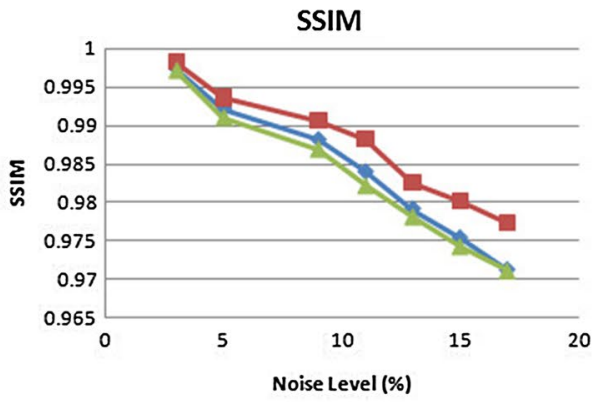

(c)

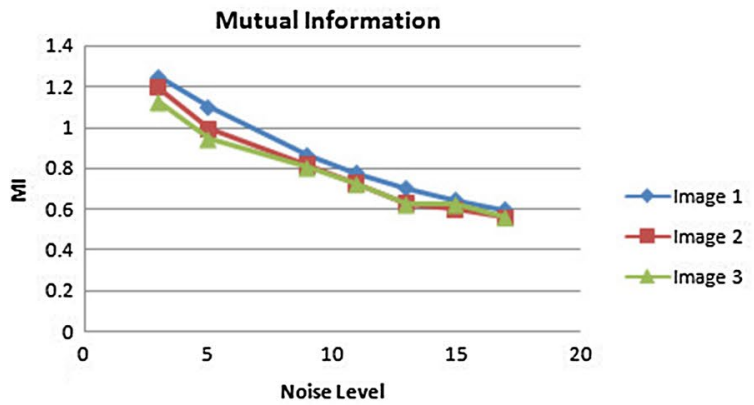

(d)

Fig. 5 a Graph of PSNR. b Graph of RMSE. c Graph of SSIM. d Graph of mutual information
4 Result analysis

\subsection{Results for different T1 weighted MRI for different noise levels}

Table 1 show that performance matrices such as PSNR, SSIM, MI, Edge Intensity and Execution time decreases and RMSE increases as noise level increases.

\subsection{Graph of PSNR, RMSE, SSIM and mutual information for three different T-2 weighted MR images at different noise level}

Graph of PSNR shows that as noise level increases, PSNR value goes on decreasing (Fig. 5).

Graph of RMSE shows that as noise level increases, RMSE value goes on increasing.

Graph of SSIM shows that as noise level increases, SSIM value goes on decreasing.

Graph of MI shows that as noise level increases, MI value goes on decreasing.

\subsection{Comparison of non local means and BM3D methods for "T-1 weighted MR image" with noise level $9 \%$}

On comparing, BM3D NIDe VST method with NLM method, we come to know that BM3D NIDe VST out perform NLM on subjective as well as objective quality metric (Fig. 6, Table 2).

\subsection{Michelson contrast}

It is measured based on max intensity and min intensity value of image. Michelson contrast value should be 1 so that image range is maintained constant. Table 3 shows that Michelson contrast increases after using CLAHE technique.

From the table, we can observe that the Michelson contrast of proposed method is higher than Michelson contrast of the BM3D-NIDe-VST method which proves that after applying CLAHE, contrast of denoised image is better than BM3D-NIDe-VST denoised image. 


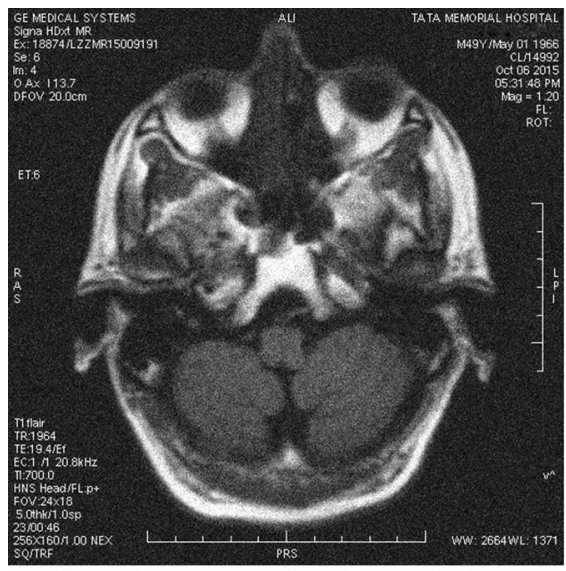

(a)

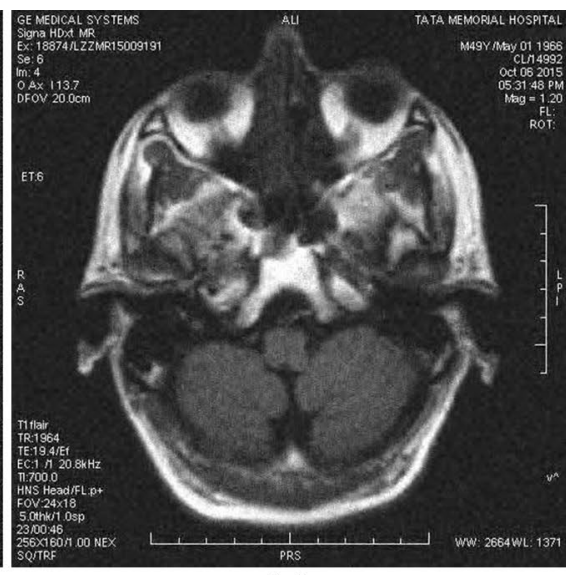

(b)

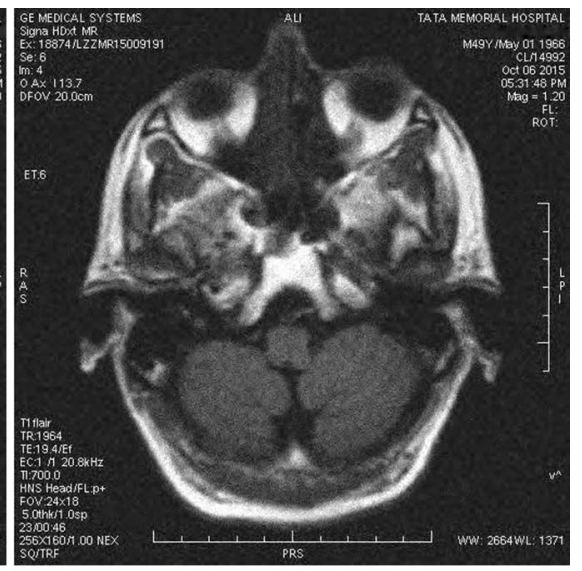

(c)

Fig. 6 a Noisy image NLM, b denoised MRI using BM3D, c denoised MRI using NLM

Table 2 Comparison table of BM3D and NLM

\begin{tabular}{llcc}
\hline Method & PSNR & SSIM & $\begin{array}{l}\text { Time } \\
\text { required } \\
\text { (min) }\end{array}$ \\
\hline BM3D & 38.0993 & 0.9866 & 4 \\
NLM & 32.8327 & 0.9821 & 34 \\
\hline
\end{tabular}

Table 3 Comparison table of BM3D-NIDe-VST with proposed method

\begin{tabular}{lll}
\hline & BM3D-NIDE-VST & $\begin{array}{l}\text { BM3D- } \\
\text { NIDE-VST- } \\
\text { CLAHE }\end{array}$ \\
\hline T-1 MRI Michelson contrast & 0.8710 & 0.9941 \\
T-2 MRI Michelson contrast & 0.8209 & 0.9948 \\
\hline
\end{tabular}

\section{Conclusion}

This Magnetic Resonance Image (MRI) denoising method consists of Block-matching and 3D filtering (BM3D) approach as well as NIDe. "Contrast limited adaptive histogram equalization (CLAHE)" is used as post-processing technique to increase the contrast of MRI. This new BM3D technique is used with the combination of NIDe, variance stabilization transform (VST) and CLAHE technique which gives image noise removal approach with improved contrast. Experiments are performed on Proton Density (PD) weighted, T1-weighted as well as on T2-weighted MR images. Performance metrics such as "Peak Signal-to-Noise ratio (PSNR)", Mutual Information (MI), "Root Mean Square Error (RMSE)" and "Structural Similarity Index Method (SSIM)" are found out. Results demonstrate advantages of the BM3D-NIDe-VST-CLAHE method over the NLM in PSNR, SSIM as well as time required for execution. Also,
Michelson Contrast is found out before CLAHE and after CLAHE shows contrast is increased. Future scope of project is that time required to execute the program can be reduced if program is executed on the parallel processing hardware.

\section{Compliance with ethical standards}

Conflict of interest All author states that there is no conflict of interest.

Ethical approval We used our own data.

Human and animal rights Humans/animals are not involved in this work.

\section{References}

1. Wright G (1997) Magnetic resonance imaging. IEEE Signal Process Mag 14:56-66

2. Henkelman RM (1985) Measurement of signal intensities in the presence of noise in MR images. Med Phys 12:232-233

3. Elahi P, Behesht S, Hashemi M (2014) BM3D MRI denoising equipped with noise invalidation technique. In: 2014 IEEE international conference on acoustic, speech and signal processing (ICASSP), pp 6612-6616

4. Mohan J, Krishnaveni V, Guo Y (2014) A survey on the magnetic resonance image denoising methods. Biomed Signal Process Control 9:56-69

5. McVeigh ER, Henkelman RM, Bronskill MJ (1985) Noise and filtration in magnetic resonance imaging. Med Phys 12:586-591

6. Krissian K, Aja-Fernández S (2009) Noise driven anisotropic diffusion filtering of MRI. IEEE Trans Image Process 18:2265-2274

7. Buades A, Coll B, Morel JM (2005) “A review of image denoising algorithms", with a new one. Multiscale Model Simul 4:490-530

8. Manjón JV, Carbonell-Caballero J, Lull JJ, García-Martí G, MartíBonmatí L, Robles M (2008) MRI denoising using non-local means. Med Image Anal 12:514-523 
9. Tomasi C, Manduchi R (1998) Bilateral filtering for gray and color images. In: Presented at the 6th int. conf. comput. vis., Bombay, pp 839-846

10. Wong WCK, Chung ACS (2004) A nonlinear and non-iterative noise reduction technique for medical images: concept and methods comparison. Int Congr Ser 1268:171-176

11. Manjon JV, Robles M, Thacker NA (2007) Multispectral MRI denoising using non-local means. In: Med. image understand. anal. (MIUA), pp 41-46

12. $\mathrm{Yu} H$, Zhao $L$ (2008) An efficient denoising procedure for magnetic resonance imaging. In: Proceedings of IEEE 2 nd international conference on bioinformatics and biomedical engineering, pp 2628-2630

13. Dabov K, Foi A, Katkovnik V, Egiazarian K (2007) Image denoising by sparse 3-D transform-domain collaborative filtering. IEEE Trans Image Process 16(8):2080-2095

14. Dabov K, Foi A, Katkovnik V, Egiazarian K (2009) BM3D image denoising with shape-adaptive principal component analysis. In: Proceedings workshop on signal processing with adaptive sparse structured representations (SPARS'09), Saint-Malo
15. Maggioni M, Katkovnik V, Egiazarian K, Foi A (2013) Nonlocal transform-domain filter for volumetric data denoising and reconstruction. IEEE Trans Image Process 22(1):119-133

16. Foi $A$ (2011) Noise estimation and removal in MR imaging: the variance-stabilization approach. In: IEEE international symposium on biomedical ımaging: from Niño to Macro, pp 1809-1814

17. Makitalo M, Foi A (2011) Optimal inversion of the Anscombe transformation in low-count Poisson image denoising. IEEE Trans Image Process 20(1):99-109

18. Beheshti S, Hashemi M, Xiao-Ping Z, Nikvand N (2010) Noise invalidation denoising. IEEE Trans Signal Process 58(12):6007-6016

19. Patil PS, Pawade PP (2016) Biomedical ımage brightness preservation and segmentation technique using CLAHE and Wiener filtering. Int J Adv Res Comput Commun Eng 5(4):808-812

Publisher's Note Springer Nature remains neutral with regard to jurisdictional claims in published maps and institutional affiliations. 